\title{
An Unusual Osteochondral Articular Surface Fracture of Second Metatarsal Head: A Case Report
}

\author{
Aytun Temiz ${ }^{\mathrm{a}, \mathrm{c}}$, Teoman Atici ${ }^{\mathrm{b}}$
}

\begin{abstract}
Osteochondral fracture involves the articular cartilage and it creates bone or cartilage fragments that can range in various size and depth. An isolated osteochondral fracture of metatarsal head is extremely rare. The treatment of these fractures is controversial. Authors present a case of an osteochondral fracture of second metatarsal head that was treated by open reduction and internal fixation with a headless compression screw.
\end{abstract}

Keywords: Osteochondral metatarsal head; Fracture; Internal fixation

\section{Introduction}

Osteochondral fracture involves the articular cartilage and it creates bone or cartilage fragments that can range in various size and depth [1]. This type of fracture usually occurs in weightbearing joints such as the knee and ankle [2]. An isolated osteochondral fracture of metatarsal head is extremely rare and a few numbers of cases have been reported [1-6]. The treatment of these fractures is controversial. Conservative and surgically treated cases with various clinical results have been reported in the literature [1-6]. Different fixation techniques were utilized in previously published surgically treated cases [1-5]. In this paper, authors present a case of an osteochondral fracture of second metatarsal head that was treated by open reduction and internal fixation with a headless compression screw.

\section{Case Report}

A 19-year-old female patient was admitted to the polyclinic

Manuscript accepted for publication January 05, 2015

aEdremit State Hospital Department of Orthopaedics and Traumatology, Balikesir, Turkey

bUniversity of Uludag Department of Orthopaedics and Traumatology, Bursa, Turkey

${ }^{\mathrm{c} C}$ Corresponding Author: Aytun Temiz, Edremit State Hospital Department of Orthopaedics, Balikesir, Turkey. Email: aytuntemiz@hotmail.com

doi: http://dx.doi.org/10.14740/jmc1738w with a history of right foot injury that occurred while she stepped down from a high ground. On landing, she hyperextended the toes and felt severe pain in her second metatarsophalangeal joint. On physical examination, swelling and ecchymosis on the forefoot was evident and the patient complained of severe pain over the second metatarsophalangeal joint. Palpation revealed tenderness, crepitation and painful motion of second metatarsophalangeal joint. Neurovascular examination was normal.

Radiographic examination of the foot showed a dorsally displaced osteochondral fragment and a bony defect on the second metatarsal head (Fig. 1).

Because of the intraarticular pattern of fracture, it was decided to perform an open anatomic reduction and compressive rigid fixation. Closed reduction was not attempted in order to protect osteochondral fragment from additional trauma.

Under spinal anesthesia, tourniquet was applied, extremity was prepared and draped. Dorsal longitudinal skin incision was made over the second metatarsophalangeal joint. Extensor tendon was retracted and the fracture was exposed with longitudinal capsulotomy. The osteochondral fragment was found to be completely separated and minimal dorsal capsular soft tissue attachment was seen. Metatarsal head was evaluated in terms of underlying bone pathology such as Frieberg disease and no evidence has found regarding such a condition. Fracture has been reduced anatomically under direct vision and provisional fixation was performed via $1 \mathrm{~mm}$ Kirschner

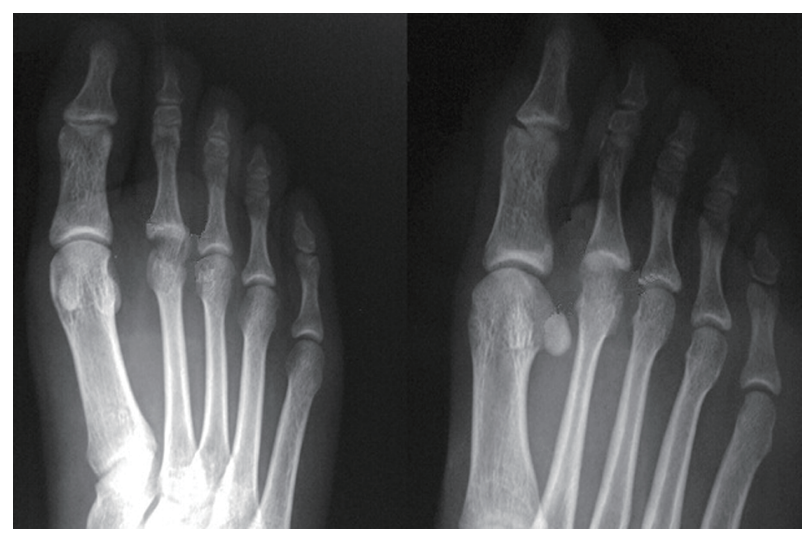

Figure 1. Preoperative X-ray of patient. Metatarsal head fracture is seen. 


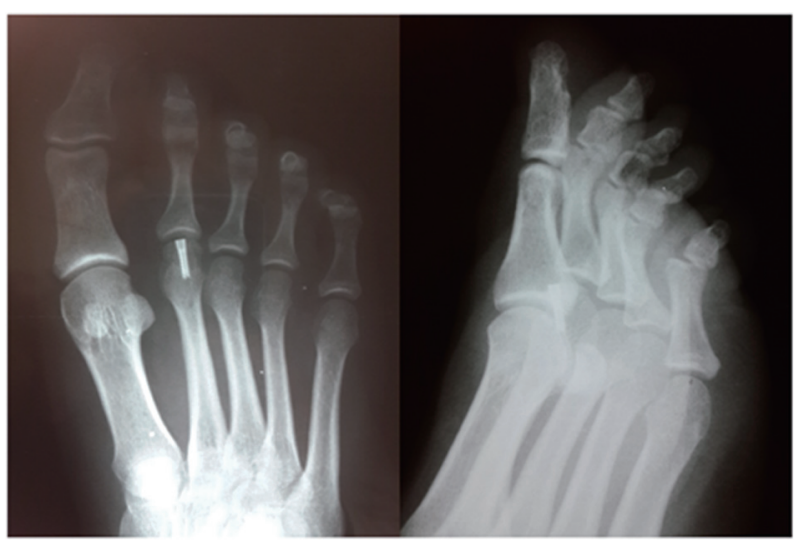

Figure 2. Early postoperative AP and lateral X-ray of the patient.

wire. During the reduction, maximum effort was performed to protect the soft tissue attachment of fracture fragment. Reduction quality was checked and permanent fixation was obtained with an $18 \mathrm{~mm}$ headless compression screw (Fig. 2). After the evaluation of joint stability and range of motion, wound was closed. A plaster cast was not applied and early active range of motion and partially weight-bearing were allowed. The patient has been followed up at regular intervals and the postoperative course was uneventful. Serial radiographs performed during follow-up visits demonstrated good screw position and progress of fracture healing. At the 12 months follow-up, the patient was asymptomatic with full range of motion of the second metatarsophalangeal joint and there was no radiographic evidence of avascular necrosis (Fig. 3).

\section{Discussion}

Isolated osteochondral fracture of the metatarsal head is a rare injury pattern [1-7]. These fractures are usually seen at the second metatarsal [1-7]. The second metatarsal is the longest and is subjected to the greatest reactive ground forces during ambulation [7]. However, osteochondral fractures of the third and fourth metatarsal head have been reported in the literature $[1,2]$.

This type of fracture can occur as a result of direct trauma or shear force on metatarsal head [1-6]. Some authors stated that direct trauma is the primary mechanism of injury $[1,6]$. However, it has been reported that shear force on metatarsal head can be the main factor rather than direct trauma [2-5]. During the toe-off phase, the base of the proximal phalanges creates compressive and shearing forces along the dorsal articular surface of the metatarsal head [7]. In our case, we think that fracture occurred as a result of shear force when her second toe was in hyperextension at the metatarsophalangeal joint.

Treatment of osteochondral fracture of the metatarsal head is controversial. Heckman [6] reported an isolated osteochondral fracture of the fourth metatarsal head which was not diagnosed initially and was not treated. This patient did well despite persistent dislocation of the metatarsal articular surface because of remodeling of the remaining metatarsal head. Dutkowsky and Freeman [2] reported a case of an osteochondral

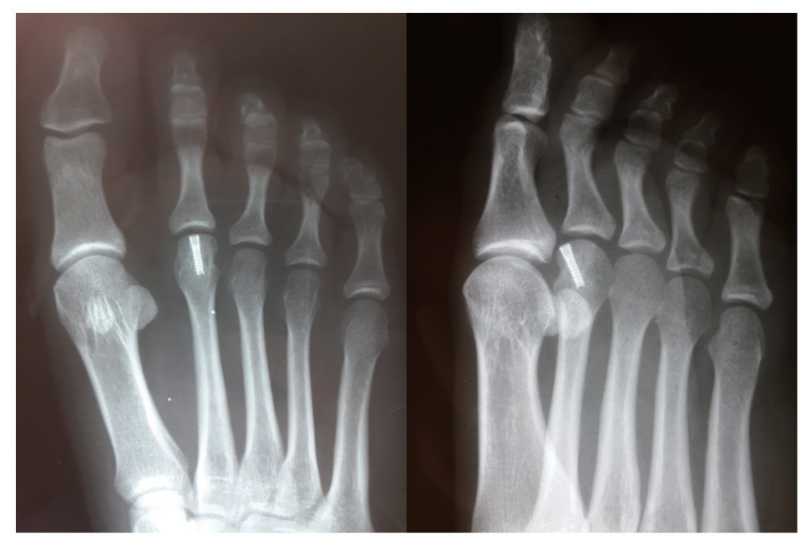

Figure 3. AP and lateral X-ray of patient at postoperative 12 months.

fracture of the third metatarsal head. They performed closed reduction and walking cast for 6 weeks. However, their patient had a loss of $10^{\circ}$ active flexion of third metatarsophalangeal joint. The final conclusion of this paper was that if closed reduction is unsuccessful, open reduction and internal fixation appears to be good alternative [2]. Liddle and Rosenfild [4] reported a case of isolated second metatarsal head fracture. Initially, conservative treatment with buddy taping and AirCast boot was attempted. After 5 months of conservative treatment, surgery was performed because of continued symptoms of unremitting pain over the second toe. The authors reported good results clinically and radiographically after surgical treatment. For this reason, they advised that early operative intervention should be performed when there is significant displacement or rotation in this type of fracture. Tanaka et al [5], Mereddy et al [1] and Atik et al [3] reported good clinical results regarding surgically treated osteochondral metatarsal head fractures. These authors initially attempted closed reduction but failed because of the small size of the displaced fracture fragment. We did not try closed reduction initially because of the prevention of osteochondral fragment from additional trauma and further fragmentation. When considering our clinical and radiologic results and aforementioned cases in the literature, it can be said that open reduction and internal fixation may be the best option for treatment of osteochondral metatarsal head fractures.

Various fixation techniques and implants have been utilized for treatment of this type of fractures [1-5]. Atik et al [3] fixed a second metatarsal head fracture via two cross K-wires and they reported excellent clinical and radiologic results. Tanaka et al [5] performed internal fixation with one Herbert screw for treatment of second metatarsal head fracture and reported good clinical and radiological results. Mereddy et al [1] used a twist-off screw for fixation of fourth metatarsal head fracture. They reported that patient was asymptomatic and there was no radiographic evidence of avascular necrosis at the end of the 12 months follow-up period. Liddle and Rosenfeld [4] also selected a $2 \mathrm{~mm}$ screw for fixation of the fracture. When considering the principles of intraarticular fracture management, we though that a headless compression screw is a good option to obtain interfragmentary compression at the fracture site.

As a conclusion, open reduction and internal fixation allow 
restoration of joint anatomy, and thus best chance of achieving normal function. However, we think that gentle handling and protection of soft tissue attachment of fracture fragment are important aspects during the operation. Because the fracture is intraarticular, rigid compressive fixation and early active postoperative motion may be the best option for treatment of this type of fracture.

\section{References}

1. Mereddy PK, Molloy A, Hennessy MS. Osteochondral fracture of the fourth metatarsal head treated by open reduction and internal fixation. J Foot Ankle Surg. 2007;46(4):320-322.

2. Dutkowsky J, Freeman BL, 3rd. Fracture-dislocation of the articular surface of the third metatarsal head. Foot An- kle. 1989;10(1):43-44.

3. Atik A, Ozyurek S, Cicek EI, Kose O. Isolated slippedretroverted osteochondral fracture of second metatarsal head. Foot (Edinb). 2013;23(4):176-179.

4. Liddle AD, Rosenfeld PF. Locked second metatarsal head fracture: a case report. Foot Ankle Int. 2008;29(10):10541056.

5. Tanaka Y, Takakura Y, Kamei S, Tamai S. An unusual osteochondral fracture of the second metatarsal head. The Foot. 1995; 5: 47-49.

6. Heckman JD. Fractures and dislocations of the foot. In: CA Rockwood Jr, D Green, JB. Fractures in Adults, 4th ed. Lippincott, Philedelphia. 1996; 2, pp 2377-2378.

7. Torriani M, Thomas BJ, Bredella MA, Ouellette H. MRI of metatarsal head subchondral fractures in patients with forefoot pain. AJR Am J Roentgenol. 2008;190(3):570575 . 